\title{
Multiple Arterial Anomalies in the Newborn Infant. Echocardiographic and Angiographic Diagnosis
}

\author{
Ivan Romero Rivera, Lourdes Gomes, Valdir Ambrósio Moisés, Celia Camelo Silva, \\ José Lázaro Andrade, Antonio Carlos Carvalho
}

São Paulo, SP - Brazil

\begin{abstract}
Multiple arterial anomalies characterized by tortuosity and rolling of the pulmonary arteries and aorta were diagnosed on echocardiography in an asymptomatic newborn infant with a phenotype suggesting EhlersDanlos syndrome. These changes were later confirmed on angiography, which also showed peripheral vascular abnormalities. The electrocardiogram showed a probable hemiblock of the left anterosuperior branch, and the chest $x$-ray showed an excavated pulmonary trunk with normal pulmonary flow.
\end{abstract}

Multiple arterial anomalies in children are rare with an almost always fatal outcome. A few cases of this malformation have been reported and its etiology remains unknown ${ }^{1}$, but its association with syndromes such as the EhlersDanlos syndrome ${ }^{2,3}$ or congenital cutis laxa ${ }^{4}$ has been mentioned. These multiple arterial anomalies may also be secondary to congenital defects of the elastic tissue of the arterial system $^{5}$.

\section{Case Report}

A full-term male newborn infant born from a normal delivery weighed 3,600 $\mathrm{g}$ and had no gestational or familial health antecedents. At the age of 28 days, he developed cough, fever, and dyspnea and was diagnosed with bronchopneumonia and atelectasis in the right pulmonary apex. The infant was hospitalized and medicated with ampicillin and amikacin. At that time, a systolic murmur (+/ $4+)$ was auscultated in the high left sternal margin, furcula, carotid artery, and dorsum. After hospital discharge, the infant was referred to our institution for ambulatory assessment.

A physical examination of the infant at the age of 38

Universidade Federal de São Paulo - Escola Paulista de Medicina

Mailing address: Antonio Carlos Carvalho - Av. Jandira, 731/23 - 04080-004 -

São Paulo, SP, Brazil

English version by Stela Maris C. Gandour days showed a body weight of 4,370 $\mathrm{g}$ and general abnormalities characterized by macrocranium, micrognathia, abduction of the thumbs, and tegumentary and ligament looseness, which became more evident with the clinical ambulatory follow-up. No edema or visceromegaly existed. The heart rate was $158 \mathrm{bpm}$, and the respiratory rate was 40 ipm. The blood pressure was $110 / 70 \mathrm{mmHg}$, and the cardiac and pulmonary auscultations were normal. The genetic evaluation stressed the clinical diagnostic hypothesis of Ehlers-Danlos syndrome.

The electrocardiogram showed sinus rhythm with a probable hemiblock of the left anterosuperior branch (electric axis at $\left.-45^{\circ}\right)$. The chest $\mathrm{x}$-ray showed levocardia with a normal-sized cardiac silhouette and pulmonary trunk excavated. The pulmonary area was normal.

An echocardiographic study was indicated and showed: situs solitus, atrioventricular and ventriculoarterial concordances, an interatrial septum with an aneurysmatic membrane of the oval fossa occluding a defect of the ostium secundum type, and no interatrial flow (fig. 1). The interventricular septum was intact, and the cardiac chambers had normal function and dimensions. The pulmonary trunk did not show the usual bifurcation, and the pulmonary arteries originated from its posterior region. Crisscrossing of the pulmonary arteries occurred: the left branch originated from the right posterior region, heading, after a short trajectory, to the left lung, while the right branch originated from the left posterior region and headed to the right lung (fig. 1). The ascending aorta showed a normal trajectory, and the arch of the aorta, which was to the left, headed to the left posterior region of the thorax, and the descending aorta could not be visualized (fig. 2). The vessels at the base of the ventricles had an anomalous trajectory and the brachiocephalic trunk had a circular trajectory before its bifurcation. The other vessels, including the left subclavian artery, showed several points of tortuosity and crisscrossing in their spiral trajectory (fig. 1). The abdominal aorta was well visualized with an evident tortuosity at the level of the diaphragm (image in siphon) 

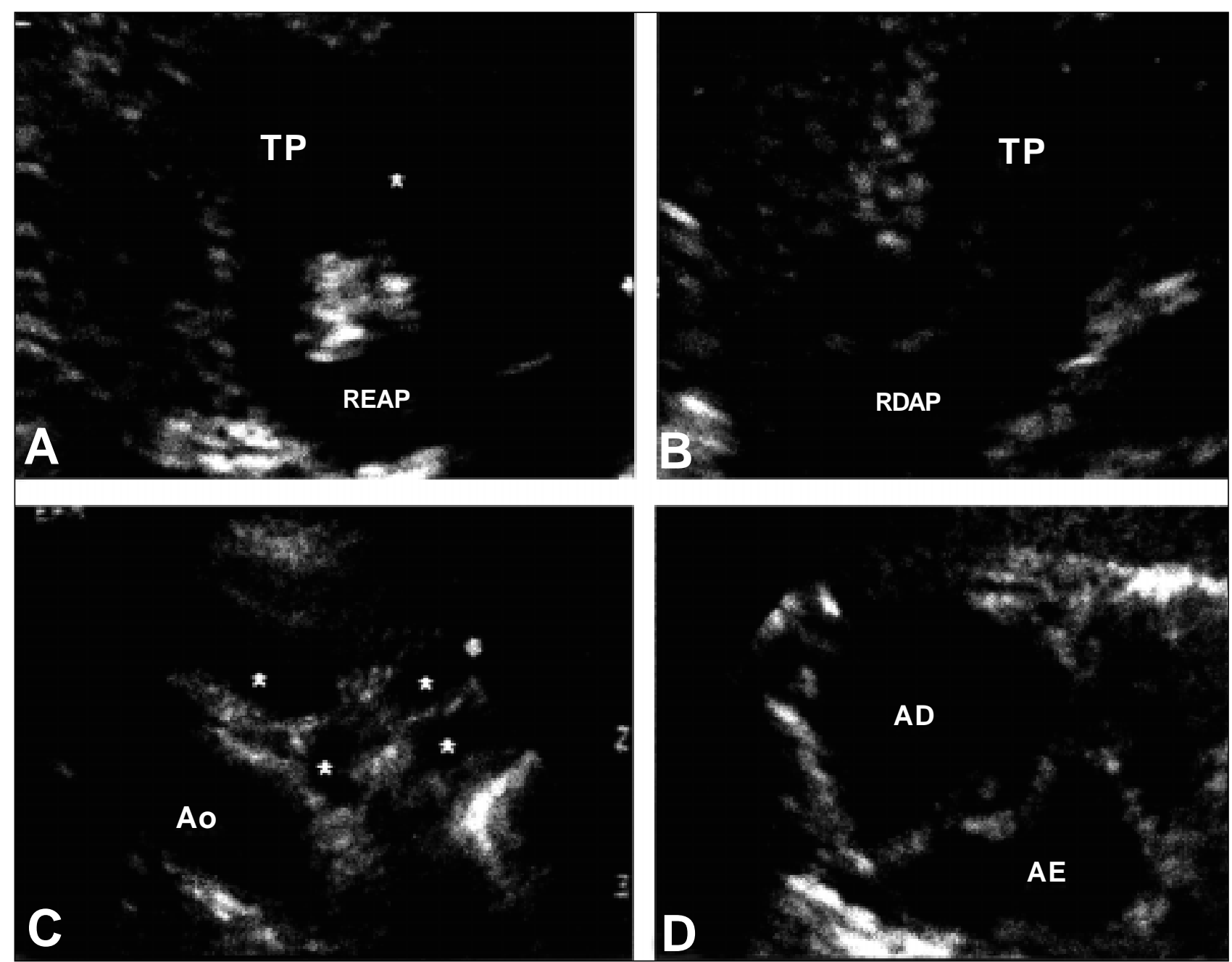

Fig. 1 - Echocardiographic data showing: A and B) crisscrossing of the pulmonary arteries in the transverse parasternal image. The asterisk in A indicates the point of origin of the RBPA. Note the cross section of the pulmonary trunk and the longitudinal section of the pulmonary arteries; C) the vessels at the base of the ventricles $(*)$ registered in the longitudinal and transverse planes in the same echocardiographic image; D) interatrial septum. RBPA - right branch of the pulmonary artery; LBPA - left branch of the pulmonary artery; Ao - aorta; PT - pulmonary trunk; RA and LA - right and left atrium, respectively.

and a retrocardiac trajectory far away from the left atrium (fig. 2).

When the infant was 11 months old, he underwent an angiographic study that confirmed the echocardiographic findings (fig. 3). After their anomalous origin, the pulmonary arteries acquired a spiral trajectory in their distal third section before their bifurcation. The arch of the aorta, to the left, gave origin to the brachiocephalic arteries, which showed tortuosity and a helicoidal rolling. After this point, the aorta headed in a transverse direction to the posterior region and stayed away from its usual position. Its descending portion, with several points of tortuosity, twisted around its own axis with a short ascending trajectory, and again it headed to the inferior region at the level of the diaphragm ("siphon" image). The coronary arteries had normal origins. The left coronary artery, however, had some points of tortuosity and multiple irregularities.

The infant was followed up on an ambulatory basis, and in his last ambulatory visit, at the age of 2 years, the patient was asymptomatic, and his physical examination was normal.

\section{Discussion}

This is an extremely rare case of echocardiographic diagnosis of multiple arterial anomalies. The angiography confirmed the echocardiographic findings and even showed other distal changes in the pulmonary arteries and the descending aorta. As far as we know, this is the first report of this type of malformation in a newborn infant. In 1858, Coulson ${ }^{6}$ reported a tortuosity of the carotid artery that was visible as a tumor in the cervical region. Reports of multiple arterial malformations are much rarer. In 1967, Ertugrul ${ }^{5}$ reported the case of a symptomatic 10-year-old child with multiple abnormalities in the aorta and its branches. No metabolic change or systemic disease was found associated with it, and Ertugrul postulated that this 

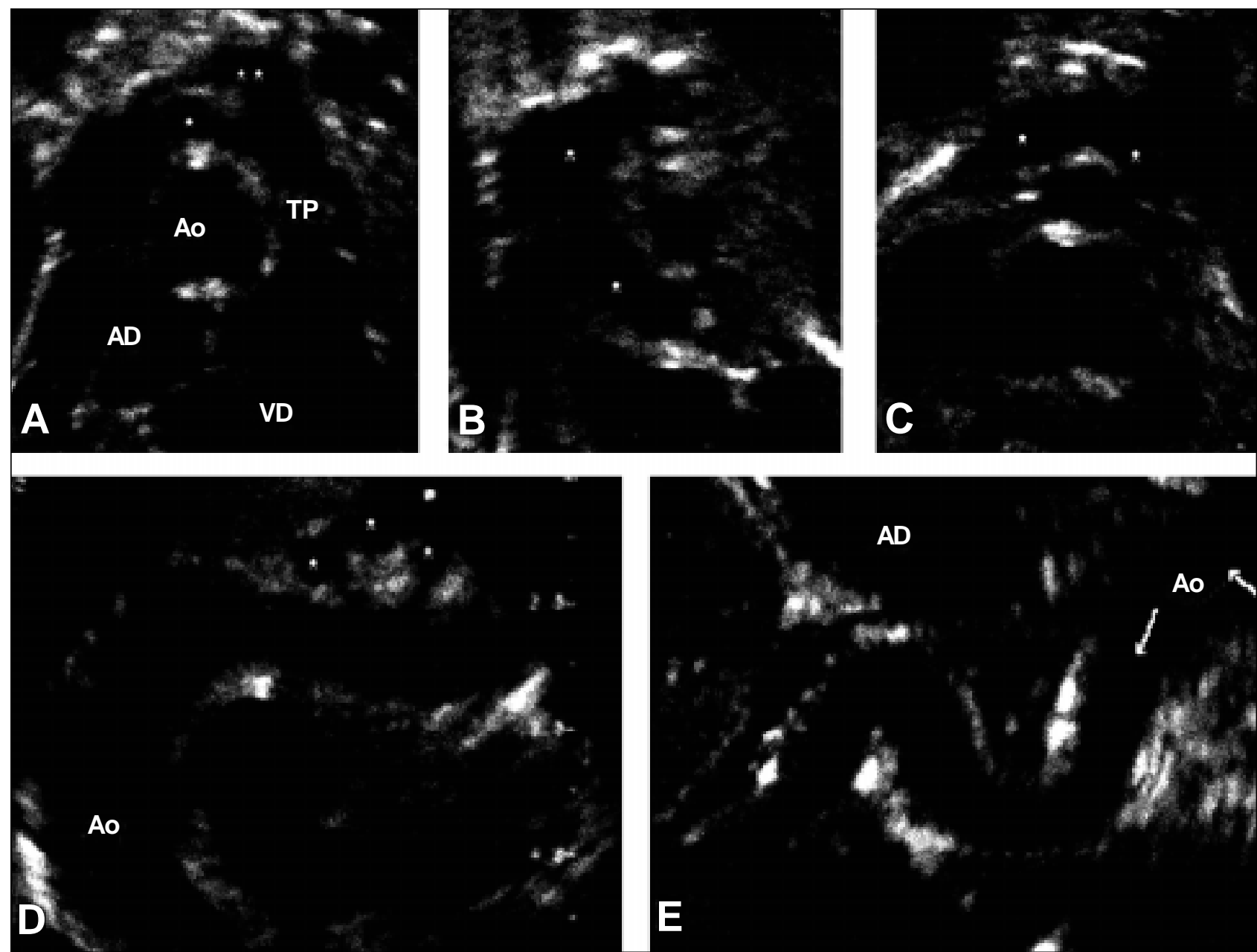

Fig. 2 - Echocardiographic data showing: A) posterior origin of the left $(*)$ and right $(*)$ pulmonary arteries in the subcostal image; B) after its origin, the LBPA runs to the left superior region heading to the corresponding lung. The RBPA appears as a circular image immediately superior; C) after its origin, the RBPA runs to the right posterior region heading to the corresponding lung. The LBPA appears as a circular image immediately inferior; D) longitudinal suprasternal image of the aorta. Note the anomalous left posterior trajectory of the descending aorta. Multiple vessels at the base of the ventricles $\left(^{*}\right)$ are seen in different planes; E) longitudinal subcostal image of the aorta. Note the anomalous trajectory in the shape of an "S". RV - right ventricle

malformation resulted from a congenital defect in the elastic tissue of the arterial system. The crisscrossing of the pulmonary arteries is also referred to as a rare entity that, in some cases, may be associated with conotruncal abnormalities or interruption of the arch of the aorta, with no other arterial changes ${ }^{7}$. Beuren et al ${ }^{1}$ reported malformations of the aorta and the pulmonary artery in a symptomatic child who died due to myocardial ischemia. This child had multiple spiral vascular changes of the carotid arteries and diffuse tortuosity in the trajectory of the vessels associated with distal stenosis of the branches, and these abnormalities were described as a new entity not related to any metabolic or systemic disease.

In our case, some data suggest an association with Ehlers-Danlos syndrome, a fact that has been previously reported ${ }^{2,3}$, mainly if we consider that the cases where the cardiovascular system is involved have no marked systemic clinical data. In a special way, type IV of the Ehlers-Danlos syndrome (Sack-Barabas) ${ }^{8}$, characterized by alterations in the synthesis of the type III collagen (present in the great vessels, skin, lung, and intestine), is associated with vascular changes. Some of these reports, however, show association with vascular aneurysms that were not found in our patient ${ }^{5,8}$.

One interesting fact in our patient is the absence of symptoms during clinical follow-up. Neurologic symptoms were reported in the presence of tortuosity of the carotid arteries in children ${ }^{9}$ or even symptoms resulting from myocardial ischemia ${ }^{1}$ due to impairment of the coronary arteries.

We stress the significant role played by echocardiography in the diagnosis of this malformation. Any change in the normal trajectory of the vessels should always be questioned, and in cases not totally defined, the angiographic confirmation is essential. 

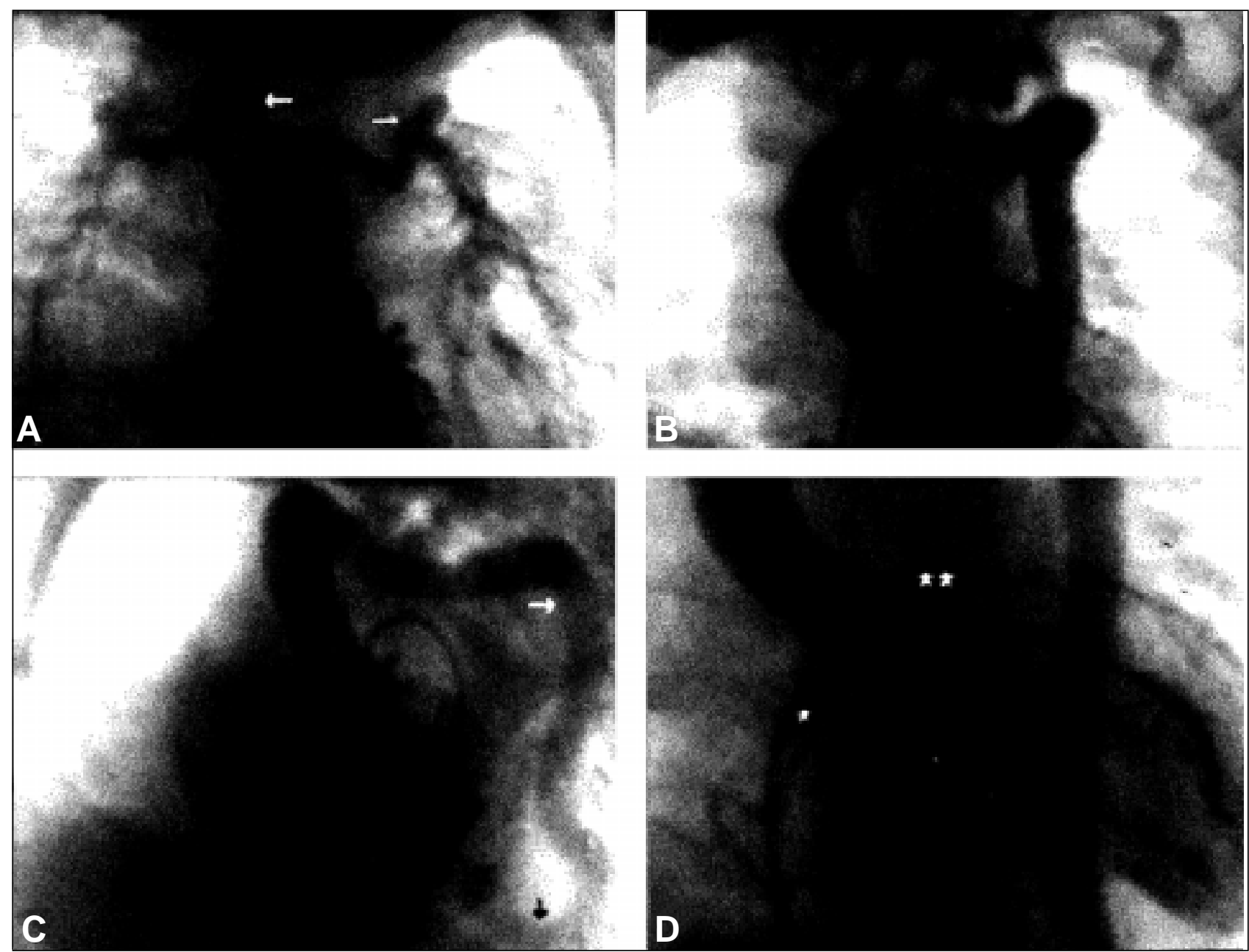

Fig. 3 - Angiographic data showing: A) anomalous origin and trajectory of the pulmonary arteries. The arrows indicate the twist point after their origin; B) anomalous direction of the arch of the aorta. The vessels at the base of the ventricles have a spiral trajectory; C) descending aorta with an anomalous trajectory and various points of tortuosity. The arrow indicates the point of tortuosity identified in the echocardiogram (fig. 2e); D) right $(*)$ and left $(* *)$ coronary arteries.

\section{References}

1. Coulson WT. Peculiar disposition of large vessels producing tumor at root neck. Trans Path Soc London 1852; 3: 302. (As cited in Ertugrul A ${ }^{6}$ ).

2.. Beuren AJ, Hort W, Kalbfleisch H, Müller H, Stoermer J. Dysplasia of the systemic and pulmonary arterial system with tortuosity and lengthening of the arteries: A new entity, diagnosed during life, and leading to coronary death in early childhood. Circulation 1969; 39: 109-15.

3. Lees MH, Menashe VD, Sunderland CO, Morgan CL, Dawson PJ. EhlersDanlos syndrome associated with multiple pulmonary artery stenoses and tortuous systemic arteries. J Pediat 1969; 75: 1031-36.

4. Imahori S, Bannerman RM, Graf CJ, Brennan JC. Ehlers-Danlos syndrome with multiple arterial lesions. Am J Med 1969; 47: 967-77.
5. Tsuji A, Yanai J, Miura T, et al. Vascular abnormalities in congenital cutis-laxa: report of two cases. Acta Paediatr Jpn 1990; 32: 155-61.

6. Ertugrul A. Diffuse tortuosity and lengthening of the arteries. Circulation 1967; 36: 400-07.

7. Wolf WJ, Casta A, Nichols M. Anomalous origin and malposition of the pulmonary arteries (crisscross pulmonary arteries) associated with complex congenital heart disease. Pediatr Cardiol 1986; 6: 287-91.

8. HunterGC,Malone JM, Moore WS, Misiorowski RL, Chvapil M. Vascularmanifestations in patients with Ehlers-Danlos syndrome. Arch Surg 1982; 117: 495-8.

9. Puzzolo D, Micali A. Embryological considerations on a multiple vascular anomaly in a child. Ital J Anat Embryol 1995; 100: 125-33. 